\title{
Engineering Disorder in Superdiffusive Lévy Glasses
}

\author{
By Jacopo Bertolotti, * Kevin Vynck, Lorenzo Pattelli, Pierre Barthelemy, \\ Stefano Lepri, and Diederik S. Wiersma*
}

Disorder is known to have a substantial impact on light transport in optical materials. In particular, when properly tuned, disorder can unveil optical properties that common, periodically patterned materials do not possess. In this paper, a method to realize disordered dielectric materials dubbed Lévy glasses, in which light transport is superdiffusive, is presented. The degree of superdiffusion is set by engineering the spatial inhomogeneity of the scatterer density in the material. A model that relates the microscopic parameters to the macroscopic transport properties of Lévy glasses is given and the signature of superdiffusion on the transmission profile in a slab configuration is shown experimentally.

\section{Introduction}

Disordered optical materials are extremely widespread in nature. From clouds to marble stone, all opaque materials owe their appearance to some random fluctuations of the refractive index. Common disordered optical materials behave as diffusers and are used, for instance, in environment lighting and solar cell technology. Disorder also plays an important role in more complex structures like photonic crystals, ${ }^{[1,2]}$ where it can strongly influence light propagation and can be engineered to obtain materials with peculiar, and potentially useful, optical properties. ${ }^{[3,4]}$

Disordered materials can be created in a variety of ways, amongst which the most simple may be that of grinding solids into fine powders. Alternative approaches include etching of glasses or semiconductors such as silicon ${ }^{[5]}$ and gallium phosphide. ${ }^{[6]}$ To engineer the scattering properties of disordered structures, one possible approach is to use monodisperse spheres and sinter them into so-called photonic glasses. ${ }^{[7]}$ The transport properties of such materials is strongly frequency-dependent, owing to the Mie resonances of each scattering elements, thereby making it possible to control, for instance, the emission spectrum of random lasers. ${ }^{[8]}$

Another approach is to modulate spatially the scatterer density. Light propagation in disordered materials can be described as a

[*] Dr. J. Bertolotti, Dr. D. S. Wiersma, Dr. K. Vynck, L. Pattelli, P. Barthelemy

European Laboratory for Nonlinear Spectroscopy (LENS) and CNRBEC

50019 Sesto-Fiorentino (FI) (Italy)

E-mail: bertolotti@lens.unifi.it; wiersma@lens.unifi.it

Dr. S. Lepri

Istituto dei Sistemi Complessi (CNR)

50019 Sesto-Fiorentino (FI) (Italy) random walk process, analogous to the Brownian motion of particles in a fluid. ${ }^{[9]}$ Having scattering elements homogeneously distributed in space generally leads to a diffusive type of transport. However, when the local density of scattering elements is position-dependent, the optical transport properties of a material can change dramatically. Therefore, one can actually modify the statistics of a random walk process in a material by engineering the distribution of the scattering elements that compose it. In particular, if the steplength distribution of the random walk is heavy-tailed, that is, falls off slowly as a power law, the diffusion approximation breaks down and the transport becomes that of a so-called Lévy walk. Lévy walks are random walk processes in which there is no upper limit to the contribution of long paths. ${ }^{[10,11]}$ As a result, the transport of Lévy walks is superdiffusive. ${ }^{[12]}$ Lévy walks are important in the most diverse fields of research, ranging from astronomy ${ }^{[13]}$ to hydrofluidics, ${ }^{[14,15]}$ ethology, ${ }^{[16]}$ and economics. ${ }^{[17]}$ Superdiffusion of light has also been observed in hot atomic vapors. ${ }^{[18]}$

In a recent paper, we have shown the first results on superdiffusion of light waves in a material dubbed Lévy glass, in which the density of the scattering elements is engineered appropriately. ${ }^{[19]}$ The early realizations of this material were based on a sodium silicate matrix. Here we discuss polymer-based Lévy glasses, in which titania particles and glass beads are dispersed in a polymer host. The use of polymer has important advantages compared to the sodium silicate, in particular regarding long-term stability. In the design of Lévy glasses, the density distribution of the scattering elements created by the size distribution of the glass beads is of fundamental importance. Thus, we will also discuss the relevant design parameters of Lévy glasses and, in particular, show how the distribution of glass beads influences the degree of superdiffusivity of these materials.

\section{Self-Assembly of Lévy Glasses}

The concept that we used to create local changes in the density of scattering elements was that of embedding them in a host medium, in which glass spheres of different sizes were incorporated (Fig. 1). Glass spheres were used for their excellent transparency for visible light and availability as near monodisperse collections with central diameters ranging from micrometers to centimeters. A relatively large set of monodisperse batches with

DOI: $10.1002 / \mathrm{adfm} .200902008$ 


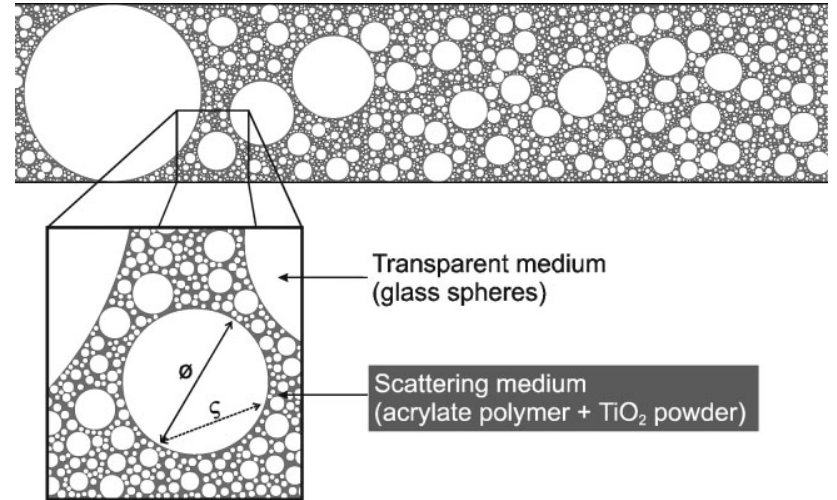

Figure 1. Representation of a 2D Lévy glass in a slab geometry, constructed from a set of 20 exponentially spaced disk diameters, spanning over two orders of magnitude. This makes the structure statistically selfsimilar over a certain range. The scattering medium (gray regions) is, by construction, forced to be present only in the interstices between the various disks (white regions). The diameter of a disk $\varnothing$ and one of the possible chords $\varsigma$ are shown in the zoomed view.

different mean diameters is actually needed to be able to reconstruct a given sphere diameter distribution. The spherical shape also avoids unnecessary complications in the modeling of the system and in the self-assembly process. As scattering elements we used $\mathrm{TiO}_{2}$ powder with sub-micrometer particle diameter, which is often used for its negligible absorption of visible light and high refractive index ( 2.7 at a wavelength of $632 \mathrm{~nm}$ ). As a host matrix we used a UV optical cure adhesive (an acrylate monomer that polymerizes upon UV light irradiation) indexmatched with the glass spheres. The use of polymers as host medium has the advantage, compared to the sodium silicate used previously, of a low shrinkage upon solidification and very good long-term stability. We tested the stability of our samples over the time span of several weeks and observed no change in their scattering properties.

The sphere diameter distribution was composed by weighting each sphere category separately and then mixing them with the titanium dioxide and the host matrix. After careful mixing, intended to avoid size clustering, the resulting viscous paste was pressed between two glass slides so that the total thickness was set by the biggest class of spheres in the sample and irradiated with UV light. The presence of the glass spheres allows UV light to reach easily the interior of the sample, despite the scattering by the $\mathrm{TiO}_{2}$ powder, and thus the structure becomes solid after just $10 \mathrm{~min}$ of irradiation.

\section{Disorder Engineering}

To obtain a Lévy walk, the step-length distribution $p(\ell)$ of the random walk process should have the form of an $\alpha$-stable Lévy distribution, ${ }^{[12]}$ typically falling off as $p(\ell) \sim \ell^{-(\alpha+1)}$ (with $0<\alpha<2$ ). In a Lévy glass, the step-length distribution is determined by the sphere diameter distribution $p(\varnothing)$. The connection between the two is important in the design of Lévy glasses. Intuitively, the fact that light should be allowed to perform steps on length scales of many orders of magnitude implies that the distribution of void sizes should also follow a power-law distribution. In the following, we show how $p(\varnothing)$ is related to $p(\ell)$, and how this sphere distribution can be implemented in practice.

After each scattering event in a Lévy glass, light travels a distance $\varepsilon$ in the scattering medium before entering a sphere, then follows a chord of the sphere, where no scattering is possible, on a distance $\varsigma$, and eventually re-enters the scattering medium. Neglecting the possibility that light will cross a second sphere before a new scattering event, we can write the conditional step-length distribution as

$p(\ell \mid \varepsilon, \varsigma)=\frac{1}{\ell_{s}}\left(H(\varepsilon-\ell) e^{-\frac{\ell}{\ell_{s}}}+H(\ell-(\varepsilon+\varsigma)) e^{-\frac{\ell-\varsigma}{\ell_{s}}}\right)$

where $H$ is the Heaviside step function and $\ell_{s}$ is the scattering mean free path in the scattering region. In the limit where spheres of infinitesimal dimension would be used and assuming near close-packing, the distance $\varepsilon$ would be vanishing small. Since, in practice, we always have lower and higher cut-offs in the sphere diameters, $\varepsilon$ is expected to be of the order of the minimal diameter $\varnothing_{0}$. Assuming that $\varepsilon \ll \varsigma$ and introducing the ansatz $p(\varsigma) \propto \varsigma^{-(\alpha+1)}$, the marginal step-length distribution becomes

$p(\ell)=\int_{0}^{\infty} p(\ell \mid \varsigma) p(\varsigma) d \varsigma \propto e^{-\ell}(\Gamma(-\alpha)-\Gamma(-\alpha,-\ell))$

where $\Gamma$ is the gamma function. An asymptotic expansion to first order of this expression shows that $p(\ell) \sim \ell^{-(\alpha+1)}$. Therefore, when $\varepsilon$ is small, the tail of $p(\ell)$ is primarily determined by that of $p(s)$.

It is important to stress the fact that $\varnothing$ and $\varsigma$ are different quantities. While the former is the diameter of a sphere, the latter represents the length of one ballistic step inside the sphere itself and is always smaller or equal to ø. The chord distribution in a sphere of diameter $\varnothing$ is given by ${ }^{[20]}$

$p(\varsigma \mid \varnothing)=\frac{(n-1)}{\varnothing^{2}} \varsigma\left(1-\frac{\varsigma^{2}}{\varnothing^{2}}\right)^{\frac{n-3}{2}} \quad(0 \leq \varsigma \leq \varnothing)$

where $n$ is the dimensionality of the system (notice that the following results are valid for any $n>1$, not only for 3D systems). We can also write the probability density function $p(\varnothing)$ to enter a sphere of diameter $\varnothing$ at each step in $n$ dimensions as

$p(\varnothing)=A \frac{n \pi^{n / 2}(\varnothing / 2)^{n-1}}{\Gamma\left(\frac{n}{2}+1\right)} \frac{1}{\varnothing^{\beta+1}}$

where $A$ is the normalization constant. The first term is the surface of the $n$-dimensional sphere, that is, how likely it is for a random point in the scattering region to be just close to the sphere, and the second term is the actual diameter distribution, which determines how many spheres with diameter $\varnothing$ are present in the sample. The choice of a power-law distribution with exponent $\beta+1$, where $\beta$ can be decided at the moment of sample 
preparation, stems from the requirement that $\ell$ should be a power law as well.

In the practical realization of a Lévy glass, one has to discretize the distribution function of the sphere diameter, since the actual sample is composed by combining known quantities of monodisperse spheres of different diameters. This means that one has to change $p(\varnothing)$ from being a continuous function to a discrete distribution of the form $p(\varnothing)=\sum p\left(\phi_{i}\right) \delta\left(\varnothing-\phi_{i}\right)$. As shown on

Figure 2, the resulting chord distribution exhibits a characteristic saw-tooth profile. This, in turn, imposes the requirement to choose the type of spacing (e.g., linear or exponential) between consecutive diameters (the sampling of the distribution function). This choice is actually of critical importance because different types of spacing at constant $\beta$ will not result in the same asymptotic behavior for $p(\varsigma)$. For instance, sets of spheres with linearly spaced diameters will present much more large spheres than those with exponentially spaced diameters, so that long steps will be significantly more important in the former case. The type of spacing can be implemented in the continuous limit by using the Riemann-Stieltjes form of the integral $p(\varsigma)=\int_{\sigma_{0}}^{\infty} p(\varsigma \mid \varnothing) p(\varnothing) d g(\varnothing)$, where $g(\varnothing)$, the integrator, maps the diameter spacing on a linear scale. When $g(\varnothing)$ is everywhere differentiable, the integral takes the form

$p(\varsigma)=\int_{\varnothing_{0}}^{\infty} p(\varsigma \mid \varnothing) p(\varnothing) \frac{\partial g(\varnothing)}{\partial \varnothing} d \varnothing$

By inserting Equations (3) and (4) into Equation (5) and taking, respectively, $g(\varnothing)=\phi^{1 / s}$ or $g(\varnothing)=\log (\varnothing)$ for polynomial spacing of power $s$ and exponential spacing, closed-form solutions to the integral are found. Recalling that $p(\varsigma) \sim \varsigma^{-(\alpha+1)}$, we finally obtain the following relations between $\alpha$ and $\beta$ in $n$-dimensional

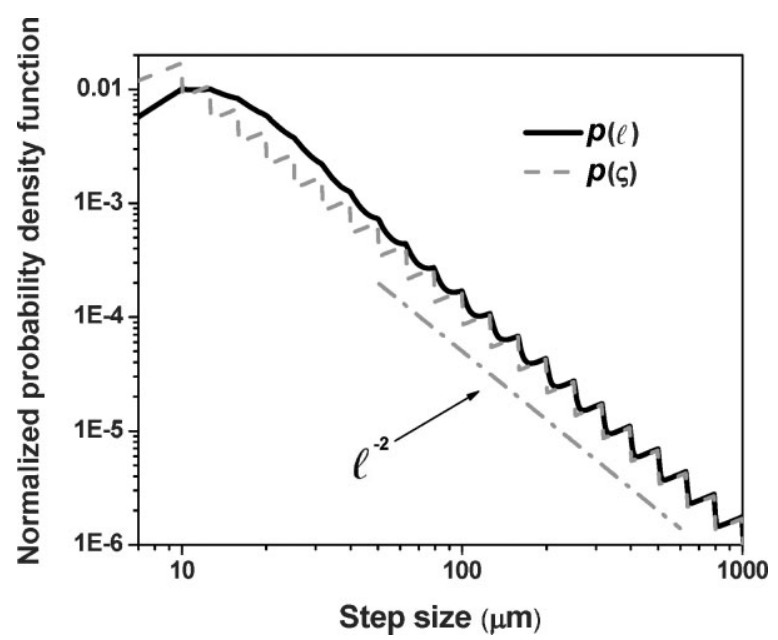

Figure 2. Comparison between $p(\varsigma)$ (gray dashed curve) and $p(\ell)$ (black curve) in a 3D Lévy glass with exponentially spaced sphere diameters and $\beta=2$. The scattering mean free path $\ell_{\mathrm{s}}$ was set to twice the smallest sphere diameter of the sample. When the diameter discretization is fine enough, $p(\ell)$ can be approximated as a power law for steps in the interval between the minimal and maximal diameters (the gray dash-dotted curve shows the slope of an exact $\ell^{-2}$ distribution as a comparison). space:

$\beta=\left\{\begin{array}{cc}\alpha+(n-2)+\frac{1}{s} & \text { polynomial } \\ \alpha+(n-2) & \text { exponential }\end{array}\right.$

Therefore, by choosing the right value of $\beta$ when we select our glass spheres, we can determine the value of $\alpha$ that characterizes the step-length distribution $p(\ell)$ and thus, control directly the degree of superdiffusivity of the material.

\section{Transmission Profile}

We realized a set of samples with $\beta$ ranging from 1.5 to 3 using 15 commercially available batches of borosilicate and soda lime glass spheres with their mean radii exponentially-spaced from 5 to $230 \mu \mathrm{m}$. This should, correspondingly, give rise to superdiffusive transport with $\alpha$ from 0.5 to 2 . In order to characterize the superdiffusive properties of each sample, we measured the spatial distribution of the light transmitted through our systems. If a sample with slab geometry is illuminated with a point-like source on one side, the distribution of intensity on the other face depends strongly on the transport properties. While in standard diffusion the main contribution to the profile is given by paths comprising a large amount of scattering events, in the case of a Lévy-like transport, paths where only one or two scattering events take place are extremely important since there is the possibility to cross the whole sample in just one jump. Therefore, while diffusive samples present a bell-shaped transmission profile, superdiffusive materials show a marked cusp on the top. In these experiments, the transmission was averaged over a large number of configurations of the disorder (see the experimental section for details). The resulting transmission curves for the various samples are plotted in Figure 3. We can clearly see how the profiles gradually change and become more cusped upon decreasing the value of $\beta$ and hence of $\alpha$. This behavior is confirmed by Monte Carlo simulations

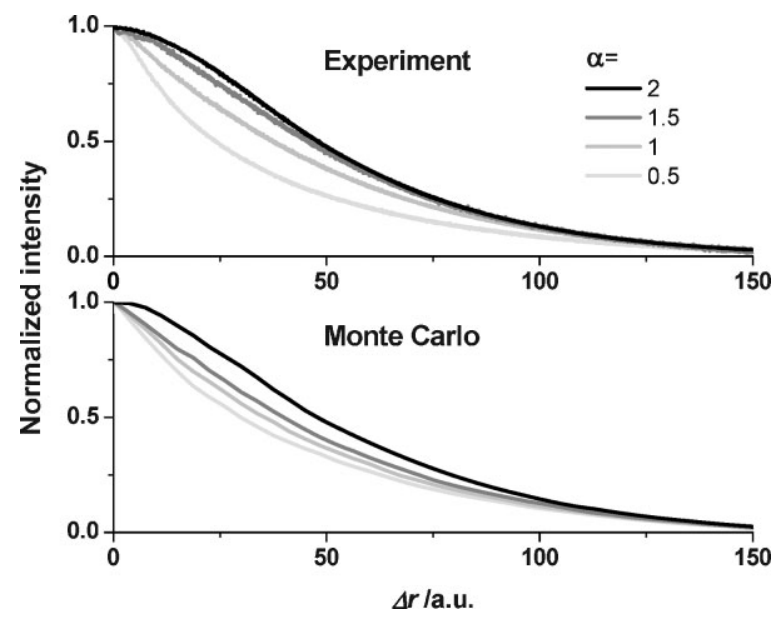

Figure 3. Upper panel: measured profiles for different sphere diameters distribution. Lower panel: Monte Carlo simulation of Lévy walks with the same nominal value of $\alpha$ as in the experiments. $\Delta r$ represents the distance from the peak of the transmission profile. 
of Lévy walks in a slab geometry, performed by using the same parameters as in the experiments, see Figure 3 for comparison.

\section{Conclusions}

We presented a simple and inexpensive recipe to realize polymerbased Lévy glasses: solid disordered optical materials where the inhomogeneities of the spatial distribution of the scattering medium, introduced in the form of glass spheres, force light to propagate superdiffusively. We discussed in details the problem of the relation between the diameter distribution (parametrized by the exponent $\beta$ ) and the step-length distribution (described by the exponent $\alpha$ ). The ability to realize samples with arbitrary $\alpha$ paves the way towards a systematic study of superdiffusion both in the coherent and in the incoherent regime and this, in turn, provides access to the study of new wave transport phenomena in disordered systems, such as, for instance, strong and weak localization and random lasing in superdiffusive media.

\section{Experimental}

The acrylate monomer used as host matrix is a commercially available optical adhesive from Thorlabs (Norlan acrylate optical adhesive 65) with refractive index 1.52 , to be compared with the refractive index of soda lime glass (1.52) and borosilicate BK7 glass (1.51). The average reflection coefficient between the spheres and the host matrix is thus smaller than $1 \%$, thereby making internal reflections negligible.

The amount of $\mathrm{TiO}_{2}$ used determines the mean free path $\ell_{\mathrm{s}}$ in the scattering region and the amount of acrylate monomer determines the total packing fraction of the glass spheres and thus $\varepsilon$ (that must be kept at minimum for the assumption $\varepsilon \ll \varsigma$ to hold). In order to have (on average) a single scattering event between any two passages across one glass sphere, we need $\ell_{s} \sim \varepsilon$. During the preparation of all the samples the total weight of the spheres was kept constant at $225 \mathrm{mg}$, and $5 \mathrm{mg}$ of $\mathrm{TiO}_{2}$ and $35 \mu \mathrm{L}$ of monomer were used.

Due to its high viscosity $(1.2 \mathrm{~Pa} \mathrm{~s})$ the monomer at room temperature cannot be efficiently mixed with the glass spheres and the $\mathrm{TiO}_{2}$. The mixing process was therefore performed while keeping the system at $150^{\circ} \mathrm{C}$ on a hot plate. The system was then pressed between two glass slides; due to the small volume of the sample this produces a quick lowering of the temperature, making the monomer highly viscous again and thus preventing any movement of the spheres on a short time-scale. Finally, after UV irradiation, the monomer polymerizes and becomes solid effectively freezing the position of all glass spheres and scattering particles.

In a real sample there must always be a lower and an upper cut-off in the diameter distribution. The smallest sphere that creates a useful void should have a diameter that is larger than the mean free path of pure $\mathrm{TiO}_{2}$ particles in the polymer host. The largest sphere, on the other hand, determines the truncation of the step length on the far end of the distribution [21] and should therefore be kept as large as possible. In practice, the largest sphere should be as large as the sample thickness.

Transmission profile measurements were performed by tightly focusing a $\mathrm{HeNe} \mathrm{cw}$ laser on one side of the sample through the glass slide and imaging with an objective the transmitted intensity distribution on a CCD camera. An average was performed over 3000 different realizations of the disorder by illuminating different regions of the same sample. The final profile was obtained by performing a radial average of the image around the maximum. In order to block the ballistic light (i.e., the fraction of light that passes through the sample without making any scattering event), the sample was placed between two crossed polarizers. Since forward scattering is almost polarization-preserving (this is rigorous for spherical or point-like particles) the use of crossed polarizers leads to an underestimation of path comprising a few (mostly forward) scattering events these paths mainly contribute to the formation of the cusp and, therefore, in the experiments, the top of the transmission profile tends to be more rounded than it should be (Fig. 3).

In the Monte Carlo simulations, the step length was generated to be distributed as the required $\alpha$-stable Lévy distribution with arbitrary value of $\alpha$ [22], while the scattering angles where chosen from a flat distribution to simulate an isotropic scattering process. Since in the experimental system, the step length has a maximum value equal to the sample thickness (i.e., the diameter of the biggest sphere present), the step-length distribution in the simulation was truncated to the same value.

\section{Acknowledgements}

The authors thank LENS for support via the project Laserlab 212025. SL aknowledges partial support by the CNR RSTL project N. 827 Dinamiche cooperative in strutture quasi uni-dimensionali. The authors thank P. D. García for useful discussion.

Received: October 26, 2009

Published online: February 22, 2010

[1] J. D. Joannopoulos, S. G. Johnson, J. N. Winn, R. D. Meade, Photonic Crystals, Molding the Flow of Light, 2nd ed., Princeton University Press, Princeton 2008.

[2] C. López, Adv. Mater. 2003, 15, 1679.

[3] S. John, Phys. Rev. Lett. 1987, 58, 2486.

[4] P. D. García, R. Sapienza, L. S. Froufe-Pérez, C. López, Phys. Rev. B. 2009, 79, 241109.

[5] O. Bisi, S. Ossicini, L. S. Pavesi, Surf. Sci. Rep. 2000, 38, 1.

[6] F. J. P. Schuurmans, D. Vanmaekelbergh, J. van de Lagemaat, A. Lagendijk, Science 1999, 284, 141

[7] P. D. García, R. Sapienza, Á. Blanco, C. López, Adv. Mater. 2007, 19, 2597.

[8] S. Gottardo, R. Sapienza, P. D. García, Á. Blanco, D. S. Wiersma, C. López, Nat. Photonics 2008, 2, 429.

[9] E. Akkermans, G. Montambaux, Mesoscopic Physics of Electrons and Photons, Cambridge University Press, Cambridge 2007.

[10] R. Klages, J. Klafter, Anomalous Transport, Wiley-VHC, Weinheim, Germany 2008

[11] R. Metzler, J. Klafter, Phys. Rep. 2000, 339, 1.

[12] J. P. Nolan, Stable Distributions, Birkhäuser, Boston, MA 2002.

[13] S. Boldyrev, C. R. Gwinn, Phys. Rev. Lett. 2003, 91, 131101.

[14] T. H. Solomon, E. R. Weeks, H. L. Swinney, Phys. Rev. Lett. 1993, 71, 3975.

[15] Y. Zhang, M. M. Meerschaert, B. Baeumer, Phys. Rev. E 2008, 78, 036705

[16] F. Bartumeus, M. G. E. Da Luz, G. M. Viswanathan, J. Catalan, Ecology 2005, 86, 3078

[17] S. Ghashghaie, W. Breymann, J. Peinke, P. Talkner, Y. Dodge, Nature 1996 $381,767$.

[18] N. Mercadier, W. Guerin, M. Chevrollier, R. Kaiser, Nat. Phys. 2009, 5, 602.

[19] P. Barthelemy, J. Bertolotti, D. S. Wiersma, Nature 2008, 453, 495.

[20] A. Mazzolo, B. Roesslinger, W. Gille, J. Math. Phys. 2003, 44, 6195.

[21] R. N. Mantegna, H. E. Stanley, Phys. Rev. Lett. 1994, 73, 2946.

[22] A. Janicki, A. Weron, Stat. Sci. 1994, 9, 109. 